\title{
Coexistence of multiple antimicrobial-resistance genes in a carbapenem-resistant Citrobacter freundii clinical isolate from China
}

Citrobacter freundii is a nosocomial pathogen associated with diarrhoea, septicaemia, meningitis, and urinary tract and respiratory system infection. Recently, there has been an increase in infections caused by multidrug-resistant $C$. freundii isolates, especially carbapenem-resistant isolates (Lee et al., 2005). Klebsiella pneumoniae carbapenemase (KPC)-type enzymes and metallo- $\beta$-lactamases are common causes of carbapenem resistance in members of the Enterobacteriaceae worldwide (Kitchel et al., 2009; Cendejas et al., 2010). KPC-2 has been found in many Enterobacteriaceae species, including C. freundii, in China (Shen et al., 2009; Zhang et al., 2008). In this report, we investigated the multiple antimicrobialresistance genes in a carbapenem-resistant C. freundii clinical isolate from a Chinese hospital. C. freundii strain NC118 was isolated from the sputum of a 23-year-old male patient hospitalized for acute lymphocytic leukaemia in April 2009 at the second affiliated hospital of Nanchang University, China. A week after admission to hospital, the patient began to cough and developed pneumonia verified by X-ray. Due to haemoptysis and lung infection, the patient died on hospital day 12. Complete biochemical identification of $C$. freundii strain NC118 was performed with the Vitek fully automated microbiology analyser system and an API 20E kit (bioMérieux). Prior to collection of the sample for culture, the patient was treated with intravenous imipenem and etimicin. C. freundii strain $\mathrm{NC1} 18$ was resistant to most clinically used antimicrobial agents, except tetracycline, as determined by disc diffusion testing according to the recommendations of the Clinical and Laboratory Standards Institute (CLSI, 2008), including ampicillin, cefoperazone, cefotaxime, ceftazidime, aztreonam, cefoxitin, imipenem, meropenem, gentamicin, tobramycin, amikacin, ciprofloxacin, levofloxacin and trimethoprim-sulfamethoxazole. MICs of antimicrobial agents determined by E-test disc diffusion were as follows $\left(\mu \mathrm{g} \mathrm{ml}^{-1}\right)$ : cefotaxime, 256; ceftazidime, 256; aztreonam, 256; cefoxitin, $>256$; imipenem, 16; meropenem, 16; gentamicin, 1024; tobramycin, 1024; amikacin, $>256$; ciprofloxacin, $>256$; trimethoprim-sulfamethoxazole, $>32$; and tetracycline, 4. PCR and DNA sequencing were used for detecting the antimicrobialresistance genes, including $\beta$-lactamase genes, plasmid-borne quinolone-resistance determinants, and 16S rRNA methylase genes. $b l a_{\mathrm{KPC}-2}$ was found in C. freundii strain NC118 by PCR and direct DNA sequencing of both strands with an $\mathrm{ABI}$ PRISM 3100 genetic analyser (Applied Biosystems). qnr genes including qnrA, $q n r B$ and $q n r S$ were determined by multiplex PCR as described previously (Robicsek et al., 2006). C. freundii strain NC118 was positive for $q n r A$ and $q n r B$. $q n r A$ - and $q n r B$-positive PCR products were sequenced and shown to match $q n r A 1$ and qnrB1. $a a c\left(6^{\prime}\right)-I b-c r$ was detected in the isolate by PCR and direct DNA sequencing (Park et al., 2006a). Extended-spectrum $\beta$-lactamase genes were screened by PCR as described previously (Yu et al., 2007). DNA sequencing of both strands was performed by the direct sequencing method. $b l a_{\mathrm{TEM}-1}$, $b l a_{\mathrm{CTX}-\mathrm{M}-3}$ and $b l a_{\mathrm{CTX}-\mathrm{M}-14}$ were found. Since C. freundii strain NC118 was highly resistant to cefoxitin (MIC $>256 \mu \mathrm{g}$ $\mathrm{ml}^{-1}$ ), plasmid-borne AmpC-lactamase genes were sought by using a multiplex PCR as described previously (Perez-Perez \& Hanson, 2002). The isolate was positive for a $b l a_{\mathrm{CMY}}$-like gene by PCR and DNA sequencing. The entire coding region of the $b l a_{\mathrm{CMY}}$-like gene was amplified with primers $b l a_{\mathrm{CMY}}-\mathrm{F}\left(5^{\prime}\right.$-CAGCAAAAATAATCAAAACCGCAAG- $\left.3^{\prime}\right)$ and $b l a_{\mathrm{CMY}}-\mathrm{R}$ (5'-GTATTGCCGGAATTACTCAAGAGCG-3'). The GenBank accession number of the sequence of the whole $1146 \mathrm{bp} b l a_{\mathrm{CMY}}{ }^{-}$ like gene obtained is GQ402541. This encoded a new CMY variant designated CMY -49 by the $\beta$-lactamase database (http://www.lahey.org/Studies/other. asp\#table1). At the protein sequence level, CMY-49 had the highest similarity of $98 \%$ (376/381) with CMY-45 (ACU00152.1). Compared with CMY-2 (PABU97164), CMY-49 has 14 amino acid substitutions. $16 \mathrm{~S}$ rRNA methylase genes, including armA, rmtA, rmtB, rmtC, rmtD and $n p m A$, were determined by PCR and DNA sequencing as described previously (Yu et al., 2009). Only armA was found in the isolate. In order to determine whether the plasmid-bearing resistance was transferable, a conjugation experiment was carried out in Luria-Bertani broth with Escherichia coli J53 as the recipient. Transconjugants were selected on tryptic soy agar plates containing sodium azide $\left(100 \mu \mathrm{g} \mathrm{ml}^{-1}\right)$ for counterselection and ampicillin $(10 \mu \mathrm{g}$ $\left.\mathrm{ml}^{-1}\right)$, ciprofloxacin $\left(0.5 \mu \mathrm{g} \mathrm{ml}^{-1}\right)$ and amikacin $\left(10 \mu \mathrm{g} \mathrm{ml}^{-1}\right)$ to select for plasmid-encoded resistance. Repeated attempts to acquire transconjugants containing plasmid-encoded resistance were unsuccessful. Plasmid DNA of $C$. freundii strain NC118 was extracted with the Qiagen Plasmid Midi kit according to the manufacturer's instructions. A

transformation experiment was used to further investigate plasmid-encoded resistance. Cefotaxime $\left(10 \mu \mathrm{g} \mathrm{ml}^{-1}\right)$, cefoxitin $\left(10 \mu \mathrm{g} \mathrm{ml}^{-1}\right)$, ciprofloxacin $\left(0.5 \mu \mathrm{g} \mathrm{ml}^{-1}\right)$ and amikacin $\left(10 \mu \mathrm{g} \mathrm{ml}^{-1}\right)$ were used for selecting transformants with plasmidencoded resistance determinants. Although the transformants harbouring quinoloneresistance determinants $b l a_{\mathrm{CTX}-\mathrm{M}}, b l a_{\mathrm{KPC}-2}$ and $\operatorname{arm} A$ were obtained, acquiring the transformant harbouring $b l a_{\mathrm{CMY}-49}$ was unsuccessful after repeated attempts. $b l a_{\mathrm{CMY}-49}$ was probably located on the chromosome of C. freundii strain NC118. The presence of class 1 integrons was determined by PCR with primers $5^{\prime} \mathrm{CS}\left(5^{\prime}\right.$ GGCATCCAAGCAGCAAGC-3') and 
3'CS (5'-AAGCAGACTTGACCTGAT-3') located in the $5^{\prime}$ and $3^{\prime}$ conserved segments. Only an approximate $2 \mathrm{~kb}$ PCR fragment was obtained from the isolate. The class 1 integron contained a $5^{\prime}$ conserved region, $d$ frA12 associated with resistance to trimethoprim, orfF, aadA2 conferring resistance to streptomycin and spectinomycin antibiotics and a $3^{\prime}$ conserved region.

$b l a_{\mathrm{KPC}-2}$ has been found coexisting with extended-spectrum $\beta$-lactamase genes and ampC in C. freundii isolates from several countries including China (Rasheed et al., 2008; Zhang et al., 2008). 16S rRNA methylase genes and plasmid-borne quinolone-resistance determinants have also been found in C. freundii isolates (Park et al., 2006b; Tamang et al., 2008). However, bla $a_{\mathrm{KPC}-2}$ coexisting with $16 \mathrm{~S}$ rRNA methylase genes and plasmid-borne quinolone-resistance determinants in the same $C$. freundii strain has not been reported before to our knowledge.

In conclusion, a total of 11 antimicrobialresistance genes, $b l a_{\mathrm{KPC}-2}, q n r A 1, q n r B 1$, $a a c\left(6^{\prime}\right)-I b-c r, b l a_{\mathrm{TEM}-1}, b l a_{\mathrm{CTX}-\mathrm{M}-3,}$ $b l a_{\mathrm{CTX}-\mathrm{M}-14}, \operatorname{armA}, \operatorname{aadA2}, d f r A 12$ and $b l a_{\mathrm{CMY}-49}$, were simultaneously detected in C. freundii strain NC118, which resulted in C. freundii strain NC118 being resistant to most of the clinically used antimicrobial agents.

\section{Acknowledgements}

This work was supported by grants from the Medical Science Fund of Zhejiang Province, China (2008A116), and Wenzhou Municipal Science and Technology Bureau, China (H2008064).

\section{Jingye Pan, ${ }^{1} \dagger$ Longhua $\mathrm{Hu}^{2}{ }^{2}$ Fangyou $\mathrm{Yu}^{3}{ }^{3}$ Chong Chen ${ }^{1}$ and Xueqing Zhang ${ }^{3}$}

${ }^{1}$ Department of Intensive Care Unit, The First Affiliated Hospital of Wenzhou
Medical College, Wenzhou 325000, PR China

${ }^{2}$ Department of Laboratory Medicine, The Second Affiliated Hospital of Nanchang University, Nanchang 336000, PR China

${ }^{3}$ Department of Laboratory Medicine, The First Affiliated Hospital of Wenzhou Medical College, Wenzhou 325000, PR China

Correspondence: Fangyou Yu (wzjxyfy@163.com)

TThese authors contributed equally to this work.

Cendejas, E., Gómez-Gil, R., Gómez-Sánchez, P. \& Mingorance, J. (2010). Detection and characterization of Enterobacteriaceae producing metallo- $\beta$-lactamases in a tertiary-care hospital in Spain. Clin Microbiol Infect 16, 181-183.

CLSI (2008). Performance Standards for Antimicrobial Susceptibility Testing, 18th Informational Supplement. M100-S18. Wayne, PA: Clinical and Laboratory Standards Institute.

Kitchel, B., Rasheed, J. K., Patel, J. B. Srinivasan, A., Navon-Venezia, S., Carmeli, Y., Brolund, A. \& Giske, C. G. (2009). Molecular epidemiology of KPC-producing Klebsiella pneumoniae isolates in the United States: clonal expansion of multilocus sequence type 258 . Antimicrob Agents Chemother 53, 3365-3370.

Lee, H. K., Park, Y. J., Kim, J. Y., Chang, E., Cho, S. G., Chae, H. S. \& Kang, C. S. (2005). Prevalence of decreased susceptibility to carbapenems among Serratia marcescens, Enterobacter cloacae, and Citrobacter freundii and investigation of carbapenemases. Diagn Microbiol Infect Dis 52, 331-336.

Park, C. H., Robicsek, A., Jacoby, G. A., Sahm, D. \& Hooper, D. C. (2006a). Prevalence in the United States of $a a c\left(6^{\prime}\right)$-Ib-cr encoding a ciprofloxacin-modifying enzyme. Antimicrob Agents Chemother 50, 3953-3955.

Park, Y. J., Lee, S., Yu, J. K., Woo, G. J., Lee, K. \& Arakawa, Y. (2006b). Co-production of $16 \mathrm{~S}$ rRNA methylases and extended-spectrum $\beta$ lactamases in AmpC-producing Enterobacter cloacae, Citrobacter freundii and Serratia marcescens in Korea. J Antimicrob Chemother 58, 907-908.

Perez-Perez, F. J. \& Hanson, N. D. (2002). Detection of plasmid-mediated AmpC $\beta$ lactamase genes in clinical isolates by using multiplex PCR. J Clin Microbiol 40,

2153-2162.

Rasheed, J. K., Biddle, J. W., Anderson, K. F., Washer, L., Chenoweth, C., Perrin, J., Newton, D. W. \& Patel, J. B. (2008). Detection of the Klebsiella pneumoniae carbapenemase type 2 carbapenem-hydrolyzing enzyme in clinical isolates of Citrobacter freundii and $\mathrm{K}$. oxytoca carrying a common plasmid. J Clin Microbiol 46, 2066-2069.

Robicsek, A., Strahilevitz, J., Sahm, D. F., Jacoby, G. A. \& Hooper, D. C. (2006). qnr prevalence in ceftazidime-resistant

Enterobacteriaceae isolates from the United States. Antimicrob Agents Chemother 50, 2872-2874.

Shen, P., Wei, Z., Jiang, Y., Du, X., Ji, S., Yu, Y. \& $\mathrm{Li}$, L. (2009). Novel genetic environment of the carbapenem-hydrolysing $\beta$-lactamase KPC-2 among Enterobacteriaceae in China. Antimicrob Agents Chemother 53, 4333-4338.

Tamang, M. D., Seol, S. Y., Oh, J. Y., Kang, H. Y., Lee, J. C., Lee, Y. C., Cho, D. T. \& Kim, J. (2008). Plasmid-mediated quinolone resistance determinants $q n r A$, $q n r B$, and $q n r S$ among clinical isolates of Enterobacteriaceae in a Korean hospital. Antimicrob Agents Chemother 52, 4159-4162.

Yu, Y., Ji, S., Chen, Y., Zhou, W., Wei, Z., Li, L. \& Ma, Y. (2007). Resistance of strains producing extended-spectrum $\beta$-lactamases and genotype distribution in China. J Infect 54, 53-57.

Yu, F., Wang, L., Pan, J., Yao, D., Chen, C., Zhu, T., Lou, Q., Hu, J., Wu, Y. \& other authors (2009). Prevalence of $16 \mathrm{~S}$ rRNA methylase genes in Klebsiella pneumoniae isolates from a Chinese teaching hospital: coexistence of $r m t B$ and $\operatorname{arm} A$ genes in the same isolate. Diagn Microbiol Infect Dis 64, 57-63.

Zhang, R., Yang, L., Cai, J. C., Zhou, H. W. \& Chen, G. X. (2008). High-level carbapenem resistance in a Citrobacter freundii clinical isolate is due to a combination of KPC-2 production and decreased porin expression. J Med Microbiol 57, 332-337. 